\title{
A Comparative Study Evaluating Improvements in Nutritional Status by Oral and Enteral Versus Parenteral Nutrition in Elderly Inpatients of an Emergency Hospital
}

\author{
Mototaka Niwano*, Kyoko Aoyama \\ General Medical Department, Kikuna Memorial Hospital, Yokohama, Japan
}

Email address:

niwano@kmh.or.jp (M. Niwano)

${ }^{*}$ Corresponding author

\section{To cite this article:}

Mototaka Niwano, Kyoko Aoyama. A Comparative Study Evaluating Improvements in Nutritional Status by Oral and Enteral Versus Parenteral Nutrition in Elderly Inpatients of an Emergency Hospital. Social Sciences. Vol. 10, No. 3, 2021, pp. 150-154.

doi: $10.11648 /$ j.ss.20211003.20

Received: May 23, 2021; Accepted: June 4, 2021; Published: June 25, 2021

\begin{abstract}
Aim: Elderly patients hospitalized in the internal medicine department who do not have sufficient oral nutritional intake and fall into low nutrition require artificial hydration and nutrition. Here, the effectiveness of total parenteral nutrition (TPN) was compared to the nutritional administration methods for discharged patients. Methods: 508 elderly inpatients who were admitted to the internal medicine department of an emergency hospital and administered TPN over 9 years were divided into two groups: patients who were discharged (331) and those who died in the hospital (177). Patients were further divided into four groups at the time of discharge from the hospital: oral nutrition, enteral nutrition, peripheral parenteral nutrition and TPN. The effectiveness of TPN was evaluated using serum albumin level and the COntrolling NUTrition Status Score (CONUT) nutritional evaluation tool. Results: While the nutritional status of patients of oral and enteral nutrition improved upon discharge, the CONUT of TPN patients without oral or enteral nutrition did not improve and serum albumin levels of the patients deteriorated. Conclusion: To discharge patients alive and safe, efforts must be made to continue oral and enteral nutrition for as long as possible.
\end{abstract}

Keywords: Elderly Inpatients, Artificial Hydration and Nutrition (AHN), Total Parenteral Nutrition (TPN), Serum Albumin Level (ALB), Controlling Nutrition Status Score (CONUT)

\section{Introduction and Purpose}

Japan currently has a multi-death super-aging society.

Elderly patients who have difficulty taking food and water orally have a low rate of $10-20 \%$ prospect of improving swallowing function [1].

The ideal nutritional administration method is oral nutrition $(\mathrm{ON})>$ enteral nutrition $(\mathrm{EN})>$ parenteral nutrition (PN) [2], and patients who have insufficient oral nutritional intake and fall into low nutrition need artificial hydration and nutrition (AHN).

AHN includes EN, peripheral $\mathrm{PN}$, and total parenteral nutrition (TPN).

EN uses intestinal ducts with percutaneous endoscopic gastrotomy (PEG) or inserts nasogastric $(\mathrm{NG})$ tube into the stomach or duodenum.
PPN refers to the intravenous infusion from the peripheral veins of limbs.

On the other hand, TPN is a central venous nutrition that uses a catheter of an intravenous drip inserted to a thick vein near the heart, such as the internal jugular vein, subclavian vein, and femoral vein, and performs a high-calorie infusion.

Our hospital, which was established in 1991 in Kohokuku, Yokohama City, Kanagawa Prefecture, has 218 hospital beds and accepts 7,000 ambulances per year, including lot of elderly inpatients.

In the actual medical field of Japanese hospitals, life prolonging treatment is performed without understanding aging death in elderly patients, and the depletion of medical resources of finance $\&$ human and medical materials matters. 
It seems that the medical staff fails to provide information on the result of life prolonging treatment of elderly patients, especially AHN.

Thus, this study aimed to concretely show the actual condition of AHN.

\section{Subjects and Method}

Elderly patients aged $\geq 65$ years as defined by the World Health Organization (WHO), who were inserted with a central venous catheter (CVC) and administered TPN were included in this study.

The patients' distribution by sex and age, classification by main disease, activities of daily life (ADL) and the food form at admission were examined.

TPN used a central venous nutrition kit formulation (Otsuka Elneopa NF No. 1 [840 cal] or No. 2 [1,230 cal] infusion, $1500 \mathrm{~mL}$, Otsuka Pharmaceutical Factory, Tokushima, Japan); for patients with renal dysfunction and hyperkalemia, handmade TPN (glucose preparation; Terumo sugar infusion, 50\%, 200mL, Terumo, Tokyo, Japan) and amino acid infusion (Neoamiyu infusion, $200 \mathrm{~mL}$, Youshindo, Toyama, Japan) with trace element formulation (ferric chloride, manganese chloride, zinc sulfate hydrate, cupric sulfate, potassium iodide combined in a $2 \mathrm{~mL}$ injection solution; Medlenik injection, Takeda Teva, Tokushima, Japan) were used.

If there were no liver functional abnormality, fatty milk (Otsuka Intraripos, 20\%, 100mL, about 275 cal, Otsuka Pharmaceutical Factory, Tokushima, Japan) was used in combination with TPN.

The concentrated fluid diet used was Isocal Support and Peptamen (Nestlé Japan, Kobe, Japan) which were administered more than $900 \mathrm{cal} /$ day.

The participants were divided into two groups: the living discharge and hospital death group. Serum albumin level (Alb), total number of lymphocytes (TLC) in the peripheral blood and total cholesterol level (T-Cho) were measured within 10 days at the time of admission and discharge and were calculated and scored using the COntrolling NUTrition Status Score (CONUT) nutritional evaluation tool (Table 1) [3].

Table 1. Assessment of undernutrition degree by CONUT Undernutrition Degree.

\begin{tabular}{lllll}
\hline Parameter & Normal & Light & Moderate & Severe \\
\hline Serum Albumin (g/dL) & $3.5-4.5$ & $3.0-3.49$ & $2.5-2.9$ & $<2.5$ \\
Score & 0 & 2 & 4 & 6 \\
Total Lymphocytes/mL & $>1600$ & $1200-1599$ & $800-1119$ & $<800$ \\
Score & 0 & 1 & 2 & 3 \\
Cholesterol (mg/dL) & $>180$ & $140-180$ & $100-139$ & $<100$ \\
Score & 0 & 1 & 2 & 3 \\
Screening Total Score & $0-1$ & $2-4$ & $5-8$ & $9-12$ \\
\hline
\end{tabular}

(Modify the diagram in Document 1)

The degree of improvement of Alb and CONUT at the time of entry and discharge from the hospital were compared among the four groups of nutritional administration method at discharge: ON, EN, PPN and TPN.

The hospital period, TPN period of each nutritional administration method, number of patients with fat milk product combination, and administration period were examined.

Statistical analyses were performed using the free software EZR [4], and the comparison among the four groups was done using the corresponding t-test, the continuous variable was analyzed using one-way analysis of variance, and the categorical variable was analyzed using chi-square test.

The group with less than six data was excluded from the test.

The significance level was set at $\mathrm{p}<0.05$.

\section{Results}

A total of 508 participants (mean age, $85.8 \pm 7.0$ years), of which 239 were males (mean age, 840 7.2 years) and 269 were females (mean age, $87.4 \pm 6.4$ years) was included in this study.

The living discharge group consisted of 331 patients (65.2\%; mean age, $85.8 \pm 6.9$ years), of which 144 were males (mean age, $83.6 \pm 7.0$ years) and 187 were females (mean age, $87.6 \pm 6.2$ years).

Meanwhile, the hospital death group consisted of 177 patients (34.8\%; mean age, $85.7 \pm 7.2$ years), of which 95 were males (mean age, $84.7 \pm 7.4$ years) and 82 were females (mean age, $86.9 \pm 6.8$ years).

The mortality rate of males and females was $39.7 \%$ and $30.5 \%$, respectively.

The patients'distribution by sex and age is summarized in Figure 1.

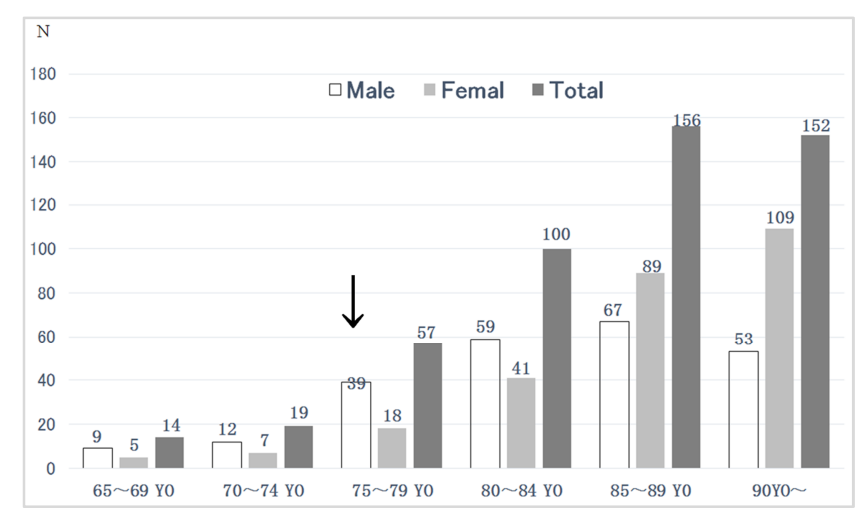

Figure 1. The patients' distribution by sex and age.

An increasing trend was shown with aging of the patients.

Regarding the main diseases, 367 (72.2\%) patients were diagnosed with pneumonia, 29 (5.7\%) patients with infection, $19(3.7 \%)$ patients with urinary tract infections, $18(3.5 \%)$ patients with digestive diseases, $16(3.2 \%)$ patients with cancer, $10(2.0 \%)$ patients with cerebral infarction, 7 (1.4\%) patients with heart failure, $7(1.4 \%)$ patients with endocrine metabolic disorders, and $35(6.9 \%)$ patients with etcetera.

ADL of hospitalized patients included 128 (25.2\%) walking patients, $94(18.5 \%)$ walking patients with a cane or 
a walker, $115(22.6 \%)$ patients on wheelchairs and 171 (33.7\%) patients requiring total assistance, showing frailty and weakening of skeletal muscle formation.

Food forms of hospitalized patients consisted of ordinary food or porridge in $240(47.3 \%)$ patients, minced meal in 113 $(22.2 \%)$, paste diet in $124(24.4 \%)$, gastrostomy in $24(4.7 \%)$, and TPN in $7(1.4 \%)$.

Elderly patients who were treated with intensive care for moderate to severe acute diseases and nutritional disorders were mostly subjected to TPN, and the ADL and food form at the time of hospitalization suggest that an advanced age and frailty were major factors of TPN administration.

The first CVC puncture sites were at the femoral veins in $410(80.7 \%)$ patients, subclavian veins in $57(11.2 \%)$, and internal jugular veins in $40(8.1 \%), 109$ patients $(21.5 \%)$ had two or more CVC insertions during hospitalization.

Regarding the nutritional administration method at discharge of the living discharge group, TPN was administered $172(52.0 \%)$ patients, ON in 110 (33.2\%), EN in $43(13.0 \%)$ and PPN in $6(1.8 \%)$.

In the 172 patients administered with TPN, seven patients had the venous port at admission and eight patients created the venous port during hospitalization.

In the 43 patients administered with EN, 17 patients PEG at admission, and 17 patients created PEG during hospitalization. There were nine patients with NG tube insertion.

Regarding the nutritional administration method at discharge of the hospital death group, TPN was administered in $165(93.2 \%)$, EN in 7 (4.0\%), and PPN in $5(2.8 \%)$.

Table 2 summarizes the number of patients according to sex, average age, period of hospital stay, TPN administration period, and number of patients who received fatty milk product, including its administration period according to the nutritional method at the time of discharge in the living discharge and hospital death groups.

Table 2. Characteristics of the living discharge and hospital death groups by nutritional administration methods the living discharge group.

\begin{tabular}{|c|c|c|c|c|}
\hline $\mathbf{N}=\mathbf{3 3 1}$ & ON 110 & EN 43 & PPN 6 & TPN 172 \\
\hline male $(\%)$ & $40(36.4 \%)$ & $21(48.8 \%)$ & $3(50 \%)$ & $80(46.5 \%)$ \\
\hline age, mean & $86.1 \pm 7.3$ & $82.7 \pm 6.2$ & $85.0 \pm 6.8$ & $86.5 \pm 6.6$ \\
\hline hospital period, mean & $40.2 \pm 20.8$ & $65.2 \pm 31.6$ & $58.3 \pm 34.9$ & $54.6 \pm 24.5^{*}$ \\
\hline TPN period, mean & $22.9 \pm 10.5$ & $32.8 \pm 12.8$ & $18.5 \pm 9.3$ & $33.3 \pm 16.5^{*}$ \\
\hline fatty milk product $(\%)$ & $107(97.3 \%)$ & $42(97.7 \%)$ & $6(100 \%)$ & $165(95.9 \%)$ \\
\hline $\begin{array}{l}\text { fatty milk product period, mean } \\
\text { the hospital death group }\end{array}$ & $20.0 \pm 11.3$ & $28.7 \pm 13.4$ & $18.2 \pm 6.3$ & $27.8 \pm 16.1^{*}$ \\
\hline $\mathrm{N}=177$ & ON 0 & EN 7 & PPN 5 & TPN 165 \\
\hline male $(\%)$ & - & $2(28.6 \%)$ & $1(20 \%)$ & $92(55.8 \%)$ \\
\hline age, mean & - & $85.6 \pm 5.4$ & $84.4 \pm 9.0$ & $85.7 \pm 7.2$ \\
\hline hospital period, mean & - & $68.1 \pm 25.6$ & $51.0 \pm 25.6$ & $37.4 \pm 24.1^{*}$ \\
\hline TPN period, mean & - & $31.7 \pm 22.3$ & $24.2 \pm 16.5$ & $20.5 \pm 15.3^{*}$ \\
\hline fatty milk product $(\%)$ & - & $7(100 \%)$ & $5(100 \%)$ & $131(79.4 \%)$ \\
\hline fatty milk product period, mean & - & $11.3 \pm 10.5$ & $20.6 \pm 18.3$ & $14.3 \pm 13.9^{*}$ \\
\hline
\end{tabular}

Comparison of TPN of the living discharge and hospital death groups *: $\mathrm{p}<0.001$

The two groups were divided into four groups (ON, EN, PPN and TPN).

For each group, the degree of Alb improvement and CONUT score at admission and discharge were examined and analyzed using the t-test (Table 3).

Table 3. Comparison of Alb and CONUT improvement according to nutritional administration method at the time of discharge - the living discharge and hospital death groups.

\begin{tabular}{|c|c|c|c|c|c|c|}
\hline & Alb admission & Alb discharge & P value & CONUT admission & CONUT discharge & P value \\
\hline \multicolumn{7}{|c|}{ the living discharge group } \\
\hline ON 110 & $3.04 \pm 0.49$ & $3.06 \pm 0.40$ & 0.652 & $6.1 \pm 2.5$ & $5.5 \pm 2.4$ & $0.025^{*}$ \\
\hline EN 43 & $2.96 \pm 0.46$ & $3.12 \pm 0.39$ & $0.043^{*}$ & $6.2 \pm 2.5$ & $4.6 \pm 2.5$ & $<0.001^{* *}$ \\
\hline PPN 6 & $2.88 \pm 0.25$ & $2.95 \pm 0.42$ & 0.833 & $6.7 \pm 2.5$ & $5.2 \pm 2.8$ & 0.279 \\
\hline $\begin{array}{l}\text { TPN } 172 \\
\text { the hospit }\end{array}$ & \multicolumn{6}{|c|}{ the hospital death group } \\
\hline EN 7 & $2.97 \pm 0.51$ & $2.74 \pm 0.37$ & $0.036^{*}$ & $5.9 \pm 2.3$ & $6.7 \pm 2.4$ & 0.17 \\
\hline
\end{tabular}

$*: \mathrm{p}<0.05, * *: \mathrm{p}<0.01$

Among the nutritional administration method in the discharged patients with TPN, the hospital period of the living discharge group and hospital death group was $54.6 \pm 24.5$ days and $37.4 \pm 24.1$ days, respectively, the TPN administration period was $33.3 \pm 16.5$ days and $20.5 \pm 15.3$ days, respectively, and the fatty milk product administration period was $27.8 \pm 16.1$ days and $14.3 \pm 13.9$ days, respectively.

A significant difference was observed between the two groups (t-test, $\mathrm{p}<0.001$; Table 2).

In the living discharge group, the nutritional administration method of TPN at discharge showed no significant difference in terms of CONUT score and Alb deterioration. In ON and 
EN administration using the intestinal system, the CONUT score was significantly improved and in EN, significant improvement of Alb was observed.

In the hospital death group, significant deterioration of $\mathrm{Alb}$ and CONUT were recognized in TPN.

Alb deterioration and no improvement of CONUT was observed in the living discharge group with TPN alone who could not be withdrawn from TPN, and it was shown that using the intestinal system was useful improving CONUT.

The living discharged patients who left TPN and recovered to $\mathrm{ON}$ and EN using the intestinal system had shortened hospital and TPN period, and the improvement of CONUT was recognized.

\section{Considerations}

A society with more than $21 \%$ of its population belonging to the elderly aged $\geq 65$ years is called a super-aging society.

Japan has entered the super-aging society in 2007 , and is currently rapidly expanding.

In September 21, 2020 (Respect for the Aged Day), 36.17 million people ( $28.7 \%$ of the total population) were aged $\geq 65$ years, indicating an incoming multi-death society.

The number of patients who died of cancer was 370,000 in 2017 , but the number of cancer deaths is expected to jump from 600,000 to 700,000 in 2020 . Including patients who die from non-cancer, the number of patient deaths exceeding 1.3 million in 2019 is expected to reach 1.6 million in 2030 .

This is also closely related to the 2025 problem, which states that in 2025 , baby boomers of large population will become the late elderly people beyond 75 years old, one in three will be over 65 years old, and one in five will be over 75 years old.

In the present study, the number of participants tended to increase with aging.

The efficacy of TPN in gastroenterological surgery and pediatrics and the risk of $\mathrm{CVC}$ infection are widely known $[5]$.

The nutritional administration method of using the intestinal system called $\mathrm{ON}$ and $\mathrm{EN}$ is physiological compared with PN.

If the small intestine is functioning, the function of the intestinal immune system is more than $50 \%$ of the whole body, and in our hospital, we are particular about eating orally.

It is currently unclear how much TPN for elderly patients hospitalized in internal medicine contributes to the improvement of nutritional status at discharge.

The subjects were divided into two groups (the living discharge and hospital death groups), and nutritional administration method at discharge was divided into four groups (ON, EN, PPN and TPN).

In this study, among $331(65.2 \%)$ of the elderly patients hospitalized with moderate to severe acute disease 153 $(46.2 \%)$ patients were safely discharged from the hospital with ON (110) and EN (43) using the intestinal system.

CONUT was significantly improved in ON and EN, and TPN seemed to be a nutritional administration method worth trying.
According to the Guidelines of The Japanese Society of Parenteral Enteral Nutrition (3rd edition, 2013)[6], the major five nutrition evaluation tools includes the following:

Subjective Global Assessment (SGA)[7], Malnutrition Universal Screening Tool (MUST, British Association for Parental Enteral Nutrition: Devised by the British Society of Venous Enteral Nutrition)[8], National Risk Score 2002 (NRS-2002)[9], Mini National Assessment (MNA)[10], and CONUT [3].

CONUT is calculated by scoring Alb, TLC and T-Cho, and it aims to a simple and low-cost nutritional evaluation tool with high effectiveness.

In CONUT, TLC is used to indicate the decrease in cell immunity due to $\mathrm{T}$ cell decrease, and $\mathrm{T}$-Cho is used to monitor appropriate nutritional administration.

Meanwhile, Alb score has twice as much weight as TLC and T-Cho.

In the present study, it was found that $\mathrm{Alb}$ deteriorated and CONUT showed no significant improvement in the living discharge group with TPN alone who could not be withdrawn from TPN.

The significant improvements in the Alb of EN and CONUT of ON and EN suggest that using the intestinal system was useful in improving Alb and CONUT.

The treatment method for elderly people who have complete difficulty in terms of oral intake is divided into four patterns $[11,12]$.

1) if patients return to the facility or home and see the situation in the natural course (oral nutrition), the remaining life is within 1 month;

2) (EN) with a life of $12-18$ months;

3) (PPN) with a life of 2-3 months;

4) (TPN) with a life of 6-12 months.

As a lot of patients have dementia, their family has to decide the method for elderly people who cannot eat and drink orally.

According to a survey on what conditions are considered terminal, $88 \%$ of old health facilities, including nursing care and geriatric health facilities, answered a relatively long period of 1-6 months at the end of life, although consent has been not made [13].

Becoming old and not eat is whether the death period or a natural providence, a biologically common process due to old age. It has not been elucidated medically.

In the super-aging with multi-death society, the effectiveness of AHN, especially TPN, was examined in this study. The results were based on the medical staff, and we hope that this study gives insights about life prolonging treatments for elderly patients.

\section{Conclusions}

Comparing the patients of the living discharge group and the hospital death group, the hospital period and TPN and fatty milk product administration period were shortened.

It was found that Alb deteriorated and CONUT showed no significant improvement in patients who discharged from the 
hospital with TPN but could not be withdrawn from TPN.

The Alb of EN and CONUT of ON and EN showed a significant improvements, indicating the usefulness of using the intestinal system in improving Alb and CONUT.

To discharged patients who are alive and safe, efforts must be made to continue oral and enteral nutrition for as long as possible.

\section{Conflict of Interest}

The authors declare that they have no competing interests.

\section{Acknowledgements}

We would like to thank the elderly patients and hospital staff who cooperated in this study. As an ethical consideration, this study obtained the approval of the ethics committee (approval number 30-03); if the patient had difficulty indicating intention, verbal consent was obtained from the family, and the patient was considered to be unidentified.

\section{References}

[1] M. Niwano, N. Murata, K. Kikuchi, et al: Examination of nutritional methods at the end of nutritional support for elderly patients. Nutrition Management Expert 2018; 15: 51-57.

[2] S. Iijima. 104th Advice, Combination pattern of intravenous nutrition and oral intake: Low nutrition to fast-paced NST rounds. Nutrition Care 2019; 12 (4): 384-389.

[3] J. Ignacio de Ulibarri, A. Gonzalez-Madrono, N. GP de Villar, et al: CONUT: A tool for Controlling Nutrition Status. First validation in a hospital population. Nutr Hosp 2005; 20: 3845.

[4] Y. Kanda. Investigation of the freely available Easy-to-use software 'EZR' for medical statistics. Bone Marrow Transplantation 2013; 48: 452-458.

[5] N. Shirotani. Parenteral Nutrition: Total Parenteral Nutrition and Peripheral Parenteral Nutrition. Japanese J Clin Physi 2006; 36: 67-73.

[6] The Japanese Society of Parenteral Enteral Nutrition. Venous Parenteral Nutrition Guidelines. $3^{\text {rd }}$ Edition, Shourinsha; 2013, 14-15.

[7] AS. Detsky, JR. McLaughlin, JP. Baker, et al: What is subjective global assessment of nutritional status? JPEN 1987; 11: 8-13.

[8] British Association for Parenteral Enteral Nutrition: Malnutrition Advisory Group (MAG). MAG-guidelines for Detection and Management of Malnutrition. Redditch, UK, 2000.

[9] J. Kondrup, SP. Allison, M. Elia, et al: ESPEN Guidelines for Nutrition Screening 2002. Clin Nutr 2003; 22: 415-421.

[10] B. Vellas B, Y. Guigoz, PJ. Garry, et al: The Mini Nutritional Assessment (MNA) and its use in grading the nutritional state of elderly patients. Nutrition 1999; 15: 116-122.

[11] T. Miyagishi, T. Higashi, Y. Akaishi, et al: Clinical features and prognosis of terminal ill patients in a long-term care hospital with particular regard to the implications of artificial nutrition. Nippon Ronen Igakkai Zasshi 2007; 44: 219-223.

[12] K. Kosaka, T. Satoh, M. Fuji, et al. Proposals for end-of-life care for the elderly. Jpn J Geriat 2008; 45: 398-40.

[13] A. Iguchi. Terminal care of the elderly. Jpn J Geriat 2005; 42: 285-287. 